This is the accepted version of the following article: Pang, J., Scrutton, N. S. and Sutcliffe, M. J. (2014), Quantum Mechanics/Molecular Mechanics Studies on the Mechanism of Action of Cofactor Pyridoxal 5'-Phosphate in Ornithine 4,5-Aminomutase. Chem. Eur. J., 20: 11390-11401.

doi: 10.1002/chem.201402759, which has been published in final form at http://dx.doi.org/10.1002/chem.201402759. 


\section{Quantum Mechanics/Molecular Mechanics Studies of the Mechanism of Action of Cofactor Pyridoxal 5'-Phosphate in Ornithine 4,5-Aminomutase}

Dr. Jiayun Pang, ${ }^{[\mathrm{a}]} *$ Prof. Nigel S. Scrutton ${ }^{[\mathrm{b}]}$ and Prof. Michael J. Sutcliffe $\mathrm{e}^{[\mathrm{c}]}$

[a] School of Science, University of Greenwich, Medway Campus, Central Avenue, Chatham Maritime, Kent, ME4 4TB, United Kingdom

[b] Manchester Institute of Biotechnology and Faculty of Life Sciences, The University of Manchester, 131 Princess Street, Manchester M1 7DN, United Kingdom.

${ }^{\text {[c] }}$ Manchester Institute of Biotechnology and School of Chemical Engineering and Analytical Science, The University of Manchester, 131 Princess Street, Manchester M1 7DN, United Kingdom.

* Dr. Jiayun Pang, Email: j.pang@gre.ac.uk, School of Science, University of Greenwich, Medway Campus, Central Avenue, Chatham Maritime, Kent, ME4 4TB, United Kingdom 


\begin{abstract}
We present here our computational study of the experimentally elusive cyclisation step in the cofactor pyridoxal 5'-phosphate (PLP)-dependent D-ornithine 4,5-aminomutase (OAM)catalysed reaction. Using both model systems and a combined QM/MM approach, our calculations suggest that regulation of the cyclic radical intermediate is achieved through the synergy of the intrinsic catalytic power of cofactor PLP and the active site of the enzyme. The captodative effect of PLP is balanced by an enzyme active site that controls the deprotonation of both the pyridine nitrogen (N1) and the Schiff base nitrogen (N2). Furthermore, electrostatic interactions between the terminal carboxylate and amine groups of the substrate and Arg297 and Glu81 impose substantial "strain" energy on the orientation of the cyclic intermediate in order to control its trajectory. In addition the "strain" energy, which appears to be sensitive to both the number of carbon atoms in the substrate/analogue and the position of the radical intermediates, may play a key role in controlling the transition of the enzyme from the closed to the open state. Our results provide new insights into several aspects of the radical mechanism in aminomutase catalysis and broaden our understanding of cofactor PLP-dependent reactions.
\end{abstract}




\section{Introduction}

Nature employs a range of cofactors to assist challenging chemical transformations in enzymes. The molecular machineries of cofactors in action are fascinating and have long intrigued enzymologists and chemists alike. Pyridoxal 5'-phosphate (PLP), the metabolically active form of Vitamin $B_{6}$, serves as cofactor in a wide variety of enzyme-catalysed reactions along the nitrogen metabolism pathway. ${ }^{[1]}$ Specifically, PLP acts as an "electron sink" to modulate intermediate formations in transamination, ${ }^{[2]}$ decarboxylation, ${ }^{[3]}$ racemisation $^{[4]}$ and elimination $^{[5]}$ reactions. These PLP-dependent enzymes are of great importance to biochemical, medicinal and biotechnological applications as they catalyse a broad range of biochemical reactions involving amino acid substrates.

In contrast to the PLP-dependent decarboxylation and transamination reactions which involve a carbanionic intermediate, PLP facilitates the repositioning of an amino group via a radical-based mechanism in ornithine 4 , 5 -aminomutase (OAM), ${ }^{[6]}$ 2,3-lysine aminomutase $(2,3-\mathrm{LAM})^{[7]}$ and 5,6-lysine aminomutase (5,6-LAM). ${ }^{[8]}$ In addition to PLP, OAM and 5,6LAM also utilise nature's radical repository - adenosylcobalamin (AdoCbl, Vitamin $\left.\mathrm{B}_{12}\right)^{[9]}$ to form radical intermediates to overcome the barrier of breaking the chemically inert $\mathrm{C}-\mathrm{H}$ and $\mathrm{C}-\mathrm{N}$ bond. The proposed catalytic cycle of $\mathrm{OAM}^{[10]}$ (Figure 1) starts with the substrate binding, triggering the homolytic rupture of the $\mathrm{Co}-\mathrm{C}$ bond to generate $\mathrm{cob}(\mathrm{II})$ alamine and the transient 5'-deoxyadenosyl radical (Ado- $\mathrm{CH}_{2} \bullet$ ), which subsequently abstracts a hydrogen from the PLP-bound substrate. This results in a PLP-bound substrate radical (CYC-1) that isomerises to form a PLP-bound product radical $(\mathrm{CYC}+1)$ via a cyclic aziridinylcarbinyl intermediate (CYC). Re-abstraction of the hydrogen from the 5'-deoxyadenosine (Ado- $\mathrm{CH}_{3}$ ) by $\mathrm{CYC}+1$ produces Ado- $\mathrm{CH}_{2}{ }^{\bullet}$, which recombines with $\mathrm{cob}(\mathrm{II})$ alamin to regenerate the AdoCbl Co-C bond.

By using a variety of computational approaches, we demonstrated recently how OAM employs a large-scale protein domain conformational change to reorientate its Rossmann domain from an open and catalytically inactive state to a closed and catalytically active state to control the generation of the transient 5 -deoxyadenosyl radical. ${ }^{[11]}$ However, the roles that PLP and the enzyme environment play to control the stability of CYC in OAM have not been fully understood. This is largely due to the difficulty of tracking the electron paramagnetic resonance (EPR) spectroscopic signals concerning CYC during steady-state turnover with OAM's natural substrate. ${ }^{[6]}$ Early quantum mechanical (QM) calculations on small model systems by Radom and co-workers ${ }^{[8 \mathrm{~h}, 10 \mathrm{~b}, 12]}$ indicated that PLP controls the relative stability of CYC through the captodative effect - the synergistic effect of the electron donating ability of the Schiff base nitrogen N2 (by its lone pair electrons) and the electron withdrawing ability of the pyridine ring (enhanced by protonation of the pyridine nitrogen N1). The captodative effect of PLP can be modulated by controlling the protonation states of N1 (via the intermolecular hydrogen bond, $\mathrm{O} 1-\mathrm{H} 1{ }^{\cdots} \mathrm{N} 1$ ) and the intramolecular hydrogen bond involving $\mathrm{N} 2(\mathrm{O} 2-\mathrm{H} 2 \cdots \mathrm{N} 2)$ (Figure 2). The hydrogen bond $\mathrm{O} 2-\mathrm{H} 2{ }^{\cdots} \mathrm{N} 2$ between the phenolic oxygen and the imine nitrogen of PLP was shown to be crucial in preventing overstabilisation of CYC.

While the theoretical studies by Radom et al have provided insights into the intrinsic nature of PLP in mediating the radical intermediates, it did not take consideration of the effect of enzymes. PLP is embedded within a hydrogen bonding network in the active site of $\mathrm{OAM},{ }^{[6 \mathrm{~d}]} 2,3-\mathrm{LAM}^{[13]}$ and 5,6-LAM. ${ }^{[8 \mathrm{~b}]}$ Despite a number of experimental mutagenesis studies, ${ }^{[6 e, 8 e]}$ the role of active site residues in controlling the reactivity and specificity of the 
radical rearrangement has not been fully understood. In the present study, we use computational studies of OAM to address a number of aspects of the radical mechanism in aminomutase catalysis.

First, the specific protonation state of the pyridine N1 and the imine N2 of PLP are critical to catalysis. ${ }^{[3 \mathrm{~b}, 14]}$ NMR experiments of PLP analogues with carboxylic acids as the hydrogen donor in $\mathrm{O} 1-\mathrm{H} 1{ }^{\cdots} \mathrm{N} 1$ showed that a proton shift from $\mathrm{O} 1$ to $\mathrm{N} 1$ in $\mathrm{O} 1-\mathrm{H} 1{ }^{\cdots} \mathrm{N} 1$ drives the tautomeric equilibrium in $\mathrm{O} 2-\mathrm{H} 2 \cdots \mathrm{N} 2$ from the neutral to the zwitterionic form. ${ }^{[15]}$ This indicates that the reactivity of the Schiff base intermediates can be tuned by differentiating the protonation state of N1. One notable difference in OAM compared to most other PLP-dependent enzymes is that N1 does not interact with the carboxylate side chain of Asp or Glu in the active site; rather it interacts with the hydroxyl group of a serine, which seemingly prohibits the protonation of N1. In addition, His225 is within hydrogen bond distance to the phenolic oxygen (O2) in the active site. Mutating it into Gln and Ala respectively lead to only 3 - and 10 -fold reduction in catalytic turnover without altering to a great extent the stability of the radical intermediates. ${ }^{[6 e]}$ We have studied whether, and how, the $\mathrm{O} 1-\mathrm{H} 1{ }^{\cdots} \mathrm{N} 1$ and $\mathrm{O} 2-\mathrm{H} 2{ }^{\cdots} \mathrm{N} 2$ hydrogen bonds modulate the radical rearrangement in OAM.

Second, highly reactive radical intermediates need to be tightly regulated by the enzymes to prevent side reactions. Both experimental and computational studies have demonstrated that the homolytic rupture of the $\mathrm{Co}-\mathrm{C}$ bond in $\mathrm{B}_{12}$-dependent enzymes and the trajectory of the newly generated Ado- $\mathrm{CH}_{2} \bullet$ are exquisitely controlled by the enzymes to ensure optimal geometry for the subsequent hydrogen $(\mathrm{H} \bullet)$ abstraction from the substrate. ${ }^{[16]}$ The interactions between the active site and the PLP-bound substrate in OAM may well play a similar role in controlling not only the energetics of CYC but also its specific orientation. Control of the geometry of the radical intermediates by the active site of OAM is studied.

Third, in OAM, the binding of its substrate D-ornithine and the substrate analogue /inhibitor D-2,4-diaminobutryic acid (DABA) both induces rapid homolysis of the AdoCbl Co-C bond. However, cob(II)alamin is a short lived intermediate that does not accumulate to detectable levels during catalytic turnover with D-ornithine. In contrast, it can be stabilised with DABA binding with strong spin-coupling to a radical intermediate derived from the PLP-bound substrate at a distance of $\sim 6$ to $7 \AA$. Consequently, the binding of DABA leads to in-activation of OAM, which probably arises from overstabilisation of a PLP-bound radical intermediate, although the exact nature of this intermediate is not clear. ${ }^{[6 \mathrm{c}]}$ Results from a recent EPR spectroscopic study ${ }^{[8 \mathrm{a}]}$ further indicate that 5,6-LAM, with D-ornithine binding, is able to switch between the open and the closed state, while it remains in the closed state with DABA binding. It is intriguing how PLP and the enzyme active site differentiate the radical species derived from D-ornithine and DABA, considering that the difference between the two is only one methylene group.

In this study, we present our computational studies of the reaction energetics and mechanism associated with the hydrogen abstraction step and more interestingly the experimentally elusive cyclisation step in OAM. Both small gas phase model systems and larger ONIOM-type combined quantum mechanical/molecular mechanical (QM/MM) systems have been used to gain insight into why radical-based aminomutase catalysis requires PLP as a cofactor and how the enzyme environment may serve to facilitate these reactions. 


\section{Results and Discussion}

Simple PLP models. To determine the effect of the protonation state/hydrogen bond of N2, three models have been constructed. The intramolecular hydrogen bond $\mathrm{O} 2-\mathrm{H} 2{ }^{\cdots} \mathrm{N} 2$ is in the neutral form in Model_1, in the zwitterionic form in Model_2, and in the neutral form but with H2 pointing away from O1 in Model_3 (Figure 2). To study the effect of the protonation of $\mathrm{N} 1$, three corresponding models were constructed: Model_1 $\left(\mathrm{H}^{+}\right)$, Model_2( $\left.\mathrm{H}^{+}\right)$and Model_3( $\left.\mathrm{H}^{+}\right)$(Figure 2). The energy and geometry features associated with CYC of the models are presented in Table 1 and Figure 3 and also in Figure S5 in the SI.

With the Schiff base N2 stabilised by the intramolecular hydrogen bond O2-H2 $\cdots \mathrm{N} 2$ (Model_1), the barrier height for ring-closing is $14.9 \mathrm{kcal} / \mathrm{mol}$ and the relative energy of CYC lies $10.2 \mathrm{kcal} / \mathrm{mol}$ above CYC-1. The barrier height for ring-opening is $18.1 \mathrm{kcal} / \mathrm{mol}$ with the energy of reaction $4.0 \mathrm{kcal} / \mathrm{mol}(\mathrm{CYC}+1)$, relative to CYC-1 $(7.9 \mathrm{kcal} / \mathrm{mol}$ and -6.2 $\mathrm{kcal} / \mathrm{mol}$ relative to CYC). In Model_2, the zwitterionic form of $\mathrm{O} 2{ }^{\cdots} \mathrm{H} 2-\mathrm{N} 2$ raises the barrier for ring-closing to $18.9 \mathrm{kcal} / \mathrm{mol}$. However, upon formation of $\mathrm{CYC}, \mathrm{O} 2{ }^{\cdots} \mathrm{H} 2-\mathrm{N} 2$ is optimised back to the neutral state during the potential energy scan to allow a path of lower potential energy to be followed. This is consistent with the radical nature of the intermediates, as deprotonation of $\mathrm{N} 2$ increases the ability of its lone pair to donate electron density to stabilise CYC. In Model_3, without the intramolecular hydrogen bond, the barrier height for ring-closing is lowered to $12.2 \mathrm{kcal} / \mathrm{mol}$ and the relative energy of CYC is also lowered to 3.6 $\mathrm{kcal} / \mathrm{mol}$. The barrier height for ring-opening is $18.2 \mathrm{kcal} / \mathrm{mol}$, relative to CYC-1.

For Model_1( $\left.\mathrm{H}^{+}\right)$, Model_2( $\left.\mathrm{H}^{+}\right)$and Model_3( $\left.\mathrm{H}^{+}\right)$, protonation of the pyridine N1 gives rise to an enhanced electron-withdrawing ability of the PLP ring. This leads to a significant reduction of the barrier height of ring-closing (TS1) in all three models. The barrier height for ring-closing is reduced from $14.9 \mathrm{kcal} / \mathrm{mol}$ to $5.9 \mathrm{kcal} / \mathrm{mol}$ (Model_1 vs Model_1 $\left(\mathrm{H}^{+}\right)$) and from $18.9 \mathrm{kcal} / \mathrm{mol}$ to $12.2 \mathrm{kcal} / \mathrm{mol}$ (Model_2 vs Model_2 $\left(\mathrm{H}^{+}\right)$). In contrast to Model_2, the zwitterionic form of $\mathrm{O} 2{ }^{\cdots} \mathrm{H} 2-\mathrm{N} 2$ is maintained in CYC in Model_2 $\left(\mathrm{H}^{+}\right)$. This is consistent with observation from the NMR studies of model PLP complexes that a proton shift from $\mathrm{O} 1$ to $\mathrm{N} 1$ drives the tautomeric equilibrium in $\mathrm{O} 2-$ $\mathrm{H} 2 \cdots \mathrm{N} 2$ from the neutral to the zwitterionic form. ${ }^{[15 \mathrm{a}]}$ The barrier height for ring-closing is also significantly reduced $(2.6 \mathrm{kcal} / \mathrm{mol})$ in Model_3( $\left.\mathrm{H}^{+}\right)$and CYC lies in a deep energy well (-11.4 kcal/mol below CYC-1 and $-17.6 \mathrm{kcal} / \mathrm{mol}$ below CYC+1).

Our results on the small model systems are in general agreement with the early computational studies by Radom and co-workers, ${ }^{[8 \mathrm{~h}, 10 \mathrm{~b}, 12 \mathrm{e}]}$ in that the barrier and the relative stability of CYC can be controlled by the protonation states of N1 and N2 in PLP. The intramolecular hydrogen bond $\mathrm{O} 2-\mathrm{H} 2{ }^{\cdots} \mathrm{N} 2$ is crucial in preventing overstabilisation of CYC, as illustrated by Model_1 versus Model_3 and Model_1 $\left(\mathrm{H}^{+}\right)$versus Model_3( $\left.\mathrm{H}^{+}\right)$. Furthermore, protonation on the pyridine N1 results in an enhanced charge delocalisation, stabilising the zwitterionic form of $\mathrm{O} 2{ }^{\cdots} \mathrm{H} 2-\mathrm{N} 2$ during cyclisation, as shown in Model_2 and Model_2 $\left(\mathrm{H}^{+}\right)$. However, the protonated N2 is incompatible with the radical nature of CYC, leading to a higher barrier for ring-closing. Thus, our gas-phase simple model results support a protonated $\mathrm{N} 1$ and a deprotonated $\mathrm{N} 2$ within the $\mathrm{O} 2-\mathrm{H} 2 \cdots \mathrm{N} 2$ hydrogen bond [i.e. Model_1 $\left(\mathrm{H}^{+}\right)$] based on its overall energy profile (Figure 3).

Extended PLP models. To study the role of the active site residues Ser162 and His225 in controlling the protonation state of N1 and N2, we extended Model_1 to include a methanol molecule to mimic the sidechain of Ser162 and an imidazole ring to represent the sidechain of His225. An acetamide molecule is also included to mimic the hydrogen bond between 
Ser162 and Gln183 (Model_4, Figure 2). It is often thought that the phosphate group of PLP only contributes to PLP binding via hydrogen bonding with the active site residues but itself plays no direct role in the mechanism. ${ }^{[1]}$ To investigate this, the phosphate group is incorporated into Model_5, and three water molecules are used to mimic the hydrogen bonds to the phosphate group within the active site.

In Model_4 and Model_5 the barrier heights for ring-closing and ring-opening and the relative energy of CYC exhibit no significant changes compared to the respective barriers in Model_1 (Table 1 and Figure 3). In Model_4, addition of the two hydrogen bonds surrounding N1 and N2 of PLP results in the barrier height of $13.5 \mathrm{kcal} / \mathrm{mol}$ for ring-closing and the barrier height of $17.0 \mathrm{kcal} / \mathrm{mol}$ for ring opening, merely $1.4 \mathrm{kcal} / \mathrm{mol}$ and 1.1 $\mathrm{kcal} / \mathrm{mol}$, respectively, below that of Model_1. In Model_5, addition of the phosphate group leads to a slight increase in the barrier for ring-closing (15.9 kcal $/ \mathrm{mol} \mathrm{cf.} 14.9 \mathrm{kcal} / \mathrm{mol})$ and a small decrease in the barrier for ring-opening (17.6 kcal/mol cf. $18.1 \mathrm{kcal} / \mathrm{mol}) \mathrm{compared}$ to Model_1. Overall, our studies indicate that the phosphate group of PLP has little impact on the energetics of the cyclisation step and the presence of the imidazole ring in the proximity of $\mathrm{O} 2-\mathrm{H} 2{ }^{\cdots} \mathrm{N} 2$ does not facilitate the deprotonation of $\mathrm{O} 2-\mathrm{H} 2$ to enhance formation of the N2-protonated zwitterionic form.

We further examined the energetics of proton transfer between $\mathrm{O} 1$ and N1 and between $\mathrm{O} 2$ and N2, respectively, using Model_4 (Figure S6 in the SI). While transfer of the hydroxyl $\mathrm{H} 1$ from $\mathrm{O} 1$ to $\mathrm{N} 1$ is associated with an energy increase of $\sim 24 \mathrm{kcal} / \mathrm{mol}$ with no clear barrier, the proton transfer from $\mathrm{N} 2$ to $\mathrm{O} 2$ has a low barrier of $\sim 3 \mathrm{kcal} / \mathrm{mol}$, with the neutral and the zwitterionic states almost in equilibrium. It has long been recognised that the tautomerisation between the N2-protonated Schiff base and the O2-protonated hydroxyimine in PLP is in a delicate equilibrium with rapid interconversion in the enzymes. There are two main factors that influence the equilibrium: the protonation state of the pyridine N1, and the substituent on the imino nitrogen of the Schiff base. ${ }^{[3 \mathrm{~b}, 4 \mathrm{~b}, 14 \mathrm{a}, 15 \mathrm{~b}, 17-18]}$ The protonated pyridine $\mathrm{N} 1$ and the carboxylate anion intermediate (as seen in majority of the PLP-dependent reactions) help stabilise the N2-protonated Schiff base. In the case of OAM, however, protonation of $\mathrm{N} 1$ via the active site serine is not favoured energetically. With $\mathrm{N} 1$ remaining neutral, a protonated $\mathrm{N} 2$ raises the barrier height of CYC formation and is incompatible with the radical nature of the intermediate.

On the other hand, Radom and co-workers observed previously that the energy requirement for radical arrangement in some $\mathrm{B}_{12}$-dependent enzymes can be reduced significantly with "partial” protonation (e.g. a hydrogen bond distance decrease of $0.05 \AA$ $\left.{ }^{[12 \mathrm{e}]}\right)$. In Model_4 and Model_5, the O1-H1 $\cdots \mathrm{N} 1$ hydrogen bond distance is decreased by $\sim 0.03 \AA$ and the O2-H2 ${ }^{\cdots} \mathrm{N} 2$ hydrogen bond distance is increased by $\sim 0.05 \AA$ in going from CYC-1 to CYC (Figure S5 in the SI). Nonetheless, the change in hydrogen bond distances does not seem to affect the barrier heights. Our model studies therefore suggest that in OAM both N1 and N2 remain neutral and deprotonated during the cyclisation step.

Combined QM/MM calculations. We used two QM regions to study the effect of the protein surrounding (Figure 4). Our preliminary QM/MM calculations indicate that the electrostatic interactions between Arg297 and Glu81 and the terminal carboxylate group and amine group of the substrate is crucial in determining the energetics of the hydrogen abstraction (Figure S3 in the SI). Thus, the QM region of QM/MM_1 includes the ribose of the AdoCbl, the PLP-bound substrate without the phosphate group and the side chains of three active site residues: Ser162, Arg297 and Glu81. QM/MM_1 is mainly used to study the energetics of the hydrogen abstraction, the formation of CYC and the hydrogen re-abstraction. 
The QM region of QM/MM_2 contains the full PLP-bound substrate, including the phosphate group and active site residues Arg109, Ser114, Tyr160, Ser162, Tyr187 and Arg192. Tyr160 and Tyr187 stack above and below the pyridine ring of PLP, forming $\pi-\pi$ interactions, whilst the side chains of Arg109, Ser114 and Arg192 form hydrogen bonds with the phosphate group of PLP. QM/MM_2 is mainly used to study the inter- and intra-molecular hydrogen bonds $\mathrm{O} 1-\mathrm{H} 1 \cdots \mathrm{N} 1$ and $\mathrm{O} 2-\mathrm{H} 2{ }^{\cdots} \mathrm{N} 2$.

The relaxed potential energy scans were first carried out with the mechanical embedding (ME) scheme. Since the ME scheme does not take into consideration the polarisation of the MM region into the QM Hamiltonian, additional single-point calculations were performed using the electronic embedding (EE) scheme, based on the ME-optimised geometry. We also performed relaxed potential energy scan using EE-optimisation for the hydrogen abstraction, cyclisation and the hydrogen re-abstraction for the PLP-bound substrate. The EE-single point calculations with the ME geometry (labelled as ME_EE) give energetics in close agreement with those from the full EE-optimisation (Table 1 and Figure S4 in the SI), and hence are used as the basis for most of the discussion.

Hydrogen abstraction from the PLP-bound substrate. The hydrogen abstraction step was studied using QM/MM_1 ${ }^{M E \_E E}$ (Figure 4). The barrier height of hydrogen abstraction from the PLP-bound substrate is $15.5 \mathrm{kcal} / \mathrm{mol}$ and the energy of reaction is $-2.5 \mathrm{kcal} / \mathrm{mol}$ (Figure 5A). The initial distance stands at $2.67 \AA$ between the transferred hydrogen and Ado- $\mathrm{CH}_{2}$ • (Figure 6A). The distance between the donor carbon and acceptor carbon was compressed to $2.75 \AA$ at the transition state with the transferred hydrogen placed equally distanced between the donor and acceptor. Upon transfer, the donor and acceptor move further apart to $3.66 \AA$. The hydrogen abstraction produces CYC-1 with spin localised at C4 (Figure S7 in the SI).

Ring-closing (CYC-1 to CYC). In CYC-1, the distance between C4 and N2 is $2.48 \AA$ $\left(\mathrm{QM} / \mathrm{MM} 1^{M E} \_E E\right.$, Figure 8). Perhaps surprisingly, formation of CYC in the enzyme requires $19.3 \mathrm{kcal} / \mathrm{mol}, 4.4 \mathrm{kcal} / \mathrm{mol}$ higher than that in Model_1 (Figure 7). CYC lies $16.5 \mathrm{kcal} / \mathrm{mol}$ above CYC-1, $6.3 \mathrm{kcal} / \mathrm{mol}$ higher than that in Model_1. It is noteworthy that other than the energetics, the orientation of the cyclic ring in CYC is also significantly different in the enzyme compared to the gas phase (Figure 9). The dihedral angle of C0-C1-N2-C4, which indicates the tilting of the ring, is $111.6^{\circ}$ in the gas phase and $132.6^{\circ}$ in $Q M / M M \_1^{M E}$ (Figure $9 \mathrm{~A}$ and Table S1 in the SI).

Decomposition of the total QM/MM energy reveals that the rise in both the barrier height and the energy of reaction is predominantly the consequence of an increase in the QM energy with only a small MM energy contribution (Figure 8(A)). We note that the MM energy here only accounts for part of the total protein effect, since Arg298 and Glu81, which form electrostatic interactions with the terminal carboxylate and amine groups of the substrate, are included in the QM region. To estimate the extent of their electrostatic effect, the "strain" energy of the PLP-bound substrate in CYC-1 and CYC is evaluated. This is carried out by comparing the energy of the PLP-bound substrate in its geometry in the enzyme and its energy optimised in the gas phase (Figure 9). The difference in energy describes how much the PLP-bound substrate is geometrically distorted in CYC-1 and CYC in the enzyme. ${ }^{[11,16 a, 19]}$ Although the energy term is usually called "strain" energy, it comprises predominantly the electrostatic effect in this case. The PLP-bound substrate in CYC-1 is subject to $20.6 \mathrm{kcal} / \mathrm{mol}$ of "strain" energy, whilst it is increased to $29.3 \mathrm{kcal} / \mathrm{mol}$ in CYC. It appears that the active site imposes increased electrostatic "strain" on the orientation of the PLP-bound substrate in going from CYC-1 to CYC, resulting in a higher barrier for CYC formation. 
The effect of intermolecular hydrogen bond $\mathrm{O} 1-\mathrm{H} 1{ }^{\cdots} \mathrm{N} 1$ on ring-closing. Within the hydrogen bonding networks between the PLP-bound substrate and the active site, a key interaction is between Ser162 and the pyridine N1. The energetics of proton transfer between the hydroxyl group of Ser162 and N1 is associated with an energy increase of $29.4 \mathrm{kcal} / \mathrm{mol}$ with no transition state in QM/MM_2 ${ }^{M E \_E E}$ (Figure S6). Therefore, protonation of N1 in PLP would not seem feasible in the enzyme.

In most PLP-dependent enzymes, the pyridine N1 is assumed to be protonated because it is hydrogen bonded to an active site Glu or Asp and the hydrogen exchange between their carboxylate side chain to $\mathrm{N} 1$ is rapid and energetically facile. ${ }^{[\mathrm{b}, 15 \mathrm{a}]}$ Other than $\mathrm{OAM}$ in which the pyridine $\mathrm{N} 1$ is hydrogen bonded to the polar serine residue, $\mathrm{N} 1$ from the PLP-dependent alanine racemase is also unprotonated, accepting a hydrogen bond from the side chain of an arginine residue. ${ }^{[20]}$ Previous computational studies of alanine racemase revealed that the protonation of $\mathrm{N} 1$ by mutating the arginine to glutamic acid may enhance the stability of the carboxylate anion intermediate and increase the likelihood of side reactions. ${ }^{[18 \mathrm{e}]}$ In addition to OAM, the pyridine N1 in 5,6-LAM is also hydrogen bonded to a serine residue, while N1 in 2,3-LAM is hydrogen bonded to a water molecule in its crystal structure. It may be beneficial for this class of radical-based aminomutase to maintain a deprotonated N1, thus increases specificity of the reaction path.

The effect of intramolecular hydrogen bond $\mathrm{O} 2-\mathrm{H} 2 \cdots \mathrm{N} 2$ on ring-closing. The proton transfer between the phenolic $\mathrm{O} 2$ and imine $\mathrm{N} 2$ in the enzyme occurs with a moderate barrier of $\sim 4.0 \mathrm{kcal} / \mathrm{mol}$ and the neutral form and the zwitterionic form in equilibrium in QM/MM_2 $2^{M E E E}$ (Figure S6). However, with the imine N2 protonated, the barrier height for the subsequent ring-closing step is increased from $24.6 \mathrm{kcal} / \mathrm{mol}$ in $\mathrm{QM} / \mathrm{MM} 2^{M E}{ }^{E E}$ to 32.9 $\mathrm{kcal} / \mathrm{mol}$ in QM/MM_2*ME_EE (Table 1 and Figure 7). In addition, similar to what is observed in Model_2, during the relaxed potential energy scan, proton H2 is transferred back to the phenolic $\mathrm{O} 2$ to return N2 to the neutral state after formation of TS1. Consistent with results from the gas phase, our QM/MM calculations rule out the possibility of N2 being protonated prior to the cyclisation step and confirm that the intramolecular $\mathrm{O} 2-\mathrm{H} 2{ }^{\cdots} \mathrm{N} 2$ hydrogen bond remains neutral.

Ring opening (CYC to CYC+1). The barrier height for ring-opening (relative to CYC) is

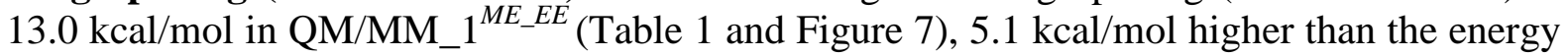
required in Model_1. The total QM energy at TS2 is decomposed into $11.3 \mathrm{kcal} / \mathrm{mol}$ of the QM energy and $1.7 \mathrm{kcal} / \mathrm{mol}$ of the MM energy. As discussed previously, the tilt of the cyclic ring is different in the enzyme than in the gas phase. In CYC +1 , the structural difference between the enzyme and gas phase model is more pronounced (Figure 9 and Table S1 in the $\mathrm{SI})$ ). The dihedral angle of $\mathrm{C} 1-\mathrm{N} 2-\mathrm{C} 4-\mathrm{C} 3$ is $96.6^{\circ}$ in the gas phase (Model_1) and is decreased to $58.5^{\circ}$ in the enzyme (QM/MM_1), which defines the position of the newly generated $\mathrm{CH}_{2}$ radical, poised to re-abstract the hydrogen from $\mathrm{AdoCH}_{3}$. Taken together with results from ring-closing, our calculations indicate that the barriers for ring-closing and opening are both lower in the gas phase, but the gas phase orientation of CYC is incompatible with the subsequent $\mathrm{H}$ re-abstraction required in the active site of OAM. The enzyme active site has to tightly control the orientation of CYC to create optimal H re-abstraction geometry, albeit at the cost of increased energy barriers.

Hydrogen re-abstraction. The hydrogen re-abstraction takes place with a barrier height of $12.8 \mathrm{kcal} / \mathrm{mol}$ and energy of reaction of $-1.2 \mathrm{kcal} / \mathrm{mol}$ (both given relative to CYC+1) in

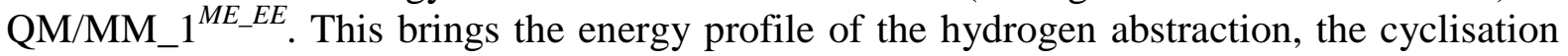
and the hydrogen re-abstraction endothermic by $15.5 \mathrm{kcal} / \mathrm{mol}$ (Figure 10). In the QM/MM 
approach used, only protein atoms within $15 \AA$ of the reaction centre are free to move. It has been hypothesised that the electrostatic interactions between Arg297 and Glu81 and the substrate may trigger the transition from the closed form to the open form in 5,6-LAM (see the next section for further discussion). If this were also the case in OAM, our potential energy based QM/MM approach may prevent incorporating the effect of larger scale protein conformational change into the cyclisation and hydrogen re-abstraction steps, thus giving an endothermic reaction profile.

Comparison with the substrate analogue DABA. Several possible radical states have been proposed for 4,5-OAM ${ }^{[6 \mathrm{c}]}$ and 5,6-LAM ${ }^{[8 \mathrm{a}]}$ to explain the mechanism of over-stabilisation of the radical intermediates following reaction with the substrate analogue DABA. We replaced the substrate ornithine in QM/MM_1 with DABA (labelled as QM/MM_DABA ${ }^{M E} E E$ ) to examine the hydrogen abstraction geometry. In the optimised structure, hydrogen abstraction from either $\mathrm{C} 3$ or C4 of the PLP-bound DABA seems feasible with an acceptor Ado- $\mathrm{CH}_{2} \bullet-$ $\mathrm{H}$ distance of $2.92 \AA$ and $2.72 \AA$, respectively (Figure 6). Our calculations indicate that abstraction of the hydrogen from C3 occurs with a barrier height of $12.6 \mathrm{kcal} / \mathrm{mol}$ and brings the system into a $-18.8 \mathrm{kcal} / \mathrm{mol}$ energy well (Figure 5). The resulting C3 radical is stabilised through spin delocalisation with the adjacent imine and pyridine ring (Figure S7 in the SI).

We have also investigated the energetics of the alternative hydrogen abstraction from C4 of the PLP-bound DABA. The barrier height is calculated to be $13.0 \mathrm{kcal} / \mathrm{mol}$ with the energy of reaction $-5.1 \mathrm{kcal} / \mathrm{mol}$ (Figure 5). A tautomer of the C4-radical formed by transfer of a proton from $\mathrm{C} 3$ to $\mathrm{C} 1^{[8 \mathrm{a}]}$ can further lower the energy of the radical to $-11.1 \mathrm{kcal} / \mathrm{mol}$ (Figure 10 and Figure S8 in the SI). Thus, both the C3-radical and the tautomer of the C4radical derived from the PLP-bound DABA are thermodynamically more stable than CYC-1 of the PLP-bound substrate $(-2.5 \mathrm{kcal} / \mathrm{mol})$. However, the calculated hyperfine coupling constants of the C3-radical are in better agreement with the experimental ENDOR data from 5,6-LAM with DABA (Table 2), which is consistent with the recent EPR study of 5,6-LAM, proposing the overstabilised C3 radical as the cause to inactivate the enzyme. ${ }^{[8]}$

The EPR study of 5,6-LAM ${ }^{[8 a]}$ also demonstrates that regardless of the radical position, analogues with an odd-number of carbon atoms (e.g. D-ornithine) could trigger transition from the closed state to the open state, whereas with analogues with an evennumber of carbon atoms (e.g. DABA) the enzyme remains in the closed state. The authors postulate $^{[8 \mathrm{a}]}$ that the conformation of the carbon atom that connects to the terminal carboxylate group and amine groups of the substrate and substrate analogues may affect transition between the closed and open states. Our calculations demonstrate that the enzyme environment imposes increased "strain" energy on the C4 radical $(22.2 \mathrm{kcal} / \mathrm{mol})$ and the C3 radical (29.6 kcal/mol) derived from the PLP-bound DABA, compared to CYC-1 of the PLPbound substrate $(20.6 \mathrm{kcal} / \mathrm{mol})$. Comparison between the "strained" conformation in the enzyme and the "relaxed" conformation in the gas phase (Figure 9) shows that the "strain" effect of the active site is reinforced by the electrostatic interactions between DABA's terminal carboxylate group and the side chain of Arg297 and between its terminal amine group and the carboxylate side chain of Glu81 (Figure 6). Optimising the conformation of CYC-1 formed within the enzyme in the gas phase (removing the "strain" energy) results in the terminal carboxylate and amine groups of the substrate flipping anti-clockwise by $\sim 60^{\circ}$ while those of DABA (the $\mathrm{C} 4$ radical) clockwise by $\sim 60^{\circ}$ (Figure $9 \mathrm{~A}$ and $9 \mathrm{~B}$ ). The conformation change of the $\mathrm{C} 3$ radical from DABA is even greater than the C4-based radicals (Figure 9C). Therefore, the electrostatic interactions between the terminal carboxylate and amine groups of the substrate and substrate analogues and the active site (Arg297 and Glu81) appear to be sensitive to the number of carbon atoms in the substrate/analogue and also the 
position of the radical intermediates. Together with the hypothesis proposed by Ke et al, ${ }^{[8 a]}$ our calculations highlight the role of the electrostatic effect at the terminal region of the substrate and substrate analogues in triggering the transition from the closed form to the open form of the enzyme.

\section{Conclusion}

In OAM, regulation of CYC is achieved through the synergistic combination of the intrinsic catalytic power of cofactor PLP and the enzyme active site. First, the captodative effect of PLP is balanced through deprotonation of both $\mathrm{N} 1$ and $\mathrm{N} 2$, which reduces electron withdrawal by the pyridine ring (compared to the N1-protonated state) and enhances electron donation by the imine N2. The deprotonated N1 serves to raise the energy barriers for ringclosing, preventing possible side reactions, while the deprotonated N2 is compatible with the radical nature of the intermediate.

Second, our calculations indicate that the dihedral angles of C0-C1-N2-C4 and C1N2-C4-C3, which define the tilting of the cyclic ring in CYC and the position of $\mathrm{CH}_{2} \bullet$ in $\mathrm{CYC}+1$, are different in the gas phase and in the enzyme. The barrier for CYC formation is lower in the gas phase, but the gas phase orientation is not compatible with the subsequent hydrogen re-abstraction in the enzyme. The role of the active site is therefore to constrain the orientation, thus controlling the trajectory of CYC to CYC+1 through electrostatic interaction with the active site Arg291 and Glu81, which creates the optimal geometry for the subsequent hydrogen re-abstraction. Consequently, the barrier height of ring-closing is increased by $4.4 \mathrm{kcal} / \mathrm{mol}$.

Third, studies of the hydrogen abstraction step between Ado- $\mathrm{CH}_{2} \bullet$ and the substrate ornithine and substrate analogue DABA, respectively, clarify the inactivation mechanisms associated with the analogue. Both C3- and C4-radical derived from the PLP-bound DABA are thermodynamically more stable. However, the EPR-detected inactivation radical species should be the C3 radical, based on the agreement between the calculated hyperfine coupling constants of C3 radical and the experimental ENDOR parameters from 5,6-LAM. ${ }^{[8 a]}$

Finally, our calculations demonstrate substantial "strain” energy arising from the electrostatic interactions between the terminal carboxylate and amine groups of the substrate and substrate analogue and the sidechains of Arg297 and Glu81. This "strain" effect may play a key part in differentiating radical species derived from the substrate and its analogues with different number of carbon atoms and consequently enable the transition between the closed state and the open state of the enzyme. In summary, our computational studies provide interesting new insights into the radical mechanism in aminomutase catalysis and more generally, broaden our understanding of cofactor PLP-dependent reactions.

\section{Experimental Section}

Gas phase model calculations. A total of eight model systems (Figure 2) were set up to investigate the effect of the protonation states of N1 and N2 in PLP. The model systems were optimised, and then relaxed potential energy scans of ring-closing (CYC-1 to CYC) and ringopening (CYC to CYC+1) were performed at both B3LYP/6-31G* and B3LYP/6-311++G** levels using program Gaussian09. ${ }^{[21]}$ Energetics obtained using the two basis sets exhibit no significant differences (Figure S9). Therefore we used B3LYP/6-31G* to represent the QM level in the subsequent QM/MM calculations, which provides a balance between the accuracy of calculations and the computational efficiency, given the number of model studied. Proton transfer between N1 and O1 and between N2 and O2 in Model_4 and Model_5 were 
computed using the more elaborate basis set $6-311++\mathrm{G}^{* *}$ for better description of the energetics of charge transfer. The minima and transition state on the potential energy surfaces were characterised by frequency calculations.

Setup of the enzyme structure. Details of the construction of the closed form of OAM and the set up of MD simulation - reported previously ${ }^{[11]}$ - are provided in the SI. In brief, the starting structure was based on the crystal structure of OAM with AdoCbl and the PLP-bound ornithine (PDB accession code: 3KOZ) and the PLP-bound DABA (3KOX), in which OAM is crystallised in its so-called open (catalytically inactive) form. The putative closed (catalytically active) form was modelled by superimposing the backbone of Rossman and TIM barrel domains of OAM, respectively, onto their counterparts in the structure of the closely related glutamate mutase (1I9C). Care was taken to adjust the conformation of AdoCbl and active site residues to avoid steric clash. The ionisable residues were modelled in the protonation state corresponding to $\mathrm{pH} 7$ obtained using programs $\mathrm{H}++(\text { version } 3.0)^{[22]}$ and PROPKA (version 3.0). ${ }^{[23]}$ The modelled structure of the closed form was then equilibrated with molecular dynamics simulations for 2 ns using the AMBER96 force field. ${ }^{[24]}$ In our QM/MM study reported previously, ${ }^{[11]}$ the QM region consisted of the corrin ring of the cobalamin, the imidazole of His618 and the Ado moiety while the MM region including residues within $\sim 20 \AA$ of the cobalt atom of AdoCbl. Residues within $\sim 15 \AA$ of the Co atom were free to move during the geometry optimisation while the rest was frozen to maintain the overall shape of the protein. The entire QM/MM system contains 8676 atoms for the closed form. The $\mathrm{Co}-\mathrm{C}$ bond was gradually elongated to a separation distance of $3.8 \AA$ during the QM/MM potential energy scan. This conformation was used as the starting point for the current QM/MM calculations.

$\mathbf{Q M} / \mathbf{M M}$ calculations. In the current $\mathrm{QM} / \mathrm{MM}$ calculations, to reduce the computational cost, the corrin ring of the cobalamin and the imidazole of His618 were moved from the QM region into the $\mathrm{MM}$ region. It should be noted that such a QM partition does not accommodate accurate inclusion of the kinetic coupling between the homolytic rupture of the Co- $\mathrm{C}$ bond and the hydrogen abstraction and the spin-spin coupling between $\mathrm{Co}(\mathrm{II})$ and the substrate/analogue radical intermediates. However, the separation distance between Co(II) and the substrate-derived radical in CYC-1 is $\sim 7 \AA$ (Figure S1(B) and Table S3 in the SI), in good agreement with the EPR-estimated range of $\sim 6-10 \AA{ }^{[6 c, 8 a]}$ The MM region comprised residues within $\sim 20 \AA$ of the cobalt atom of AdoCbl, with residues within $\sim 15 \AA$ of the Co atom free to move and the rest frozen during the geometry optimisation to maintain the shape of the protein scaffold. This partition of the "frozen" and "free" MM regions is illustrated in Figure S1 of the SI.

To study the hydrogen abstraction and the cyclisation step in the closed form of OAM, combined QM/MM relaxed potential energy scans were carried out with a two-layer $\mathrm{ONIOM}^{[25]}$ scheme, as implemented in Gaussian09, with both the mechanical embedding (ME) and the electronic embedding (EE) scheme. The B3LYP functional and 6-31G* basis set and the AMBER96 force field were employed to represent the QM and the MM regions, respectively. The two QM regions comprised 87 and 97 atoms, respectively, incorporating the Ado moiety, the PLP-bound substrate and key active site residues (Figure 4). For each series of relaxed potential energy scan, an initial optimisation was performed without constraint on the reaction coordinate to locate a minimum on the reactant side. Then, a series of constrained optimisations were performed, one by one, with the reaction coordinate constrained to move to the product side. This is followed by an unconstrained optimisation to relax the system to a minimum on the product side to complete the scan. Thus, the starting structure (the PLP-bound D-ornithine or DABA) and the intermediates (CYC-1, CYC and 
$\mathrm{CYC}+1$ ) are all subject to unconstrained QM/MM optimisation (aside from the potential constraints on the "frozen" MM region) and occupy minima on the potential energy profile. Due to the size of the systems (8676 atoms), we were not able to perform optimisation to locate transition states, nor were we able to perform frequency calculations to verify minima and transition states on the potential energy surfaces. Instead, the reaction coordinate is varied by a smaller step size in each optimisation near the top of potential barrier to locate the highest point. The EPR parameters of the deuterium at C1 of the PLP-bound DABA were computed at the B3LYP/6-311G(2d,p) level, which has been shown to be adequate in characterising PLP-bound radicals. ${ }^{[\mathrm{a},}{ }^{10 \mathrm{~b}]}$ The resulting hyperfine coupling constants displayed in Table 2 is the sum of the anisotropic component $\left(T_{i j}\right)$ and the isotropic component $\left(A_{\text {iso }}\right)$.

\section{Acknowledgements}

This work was funded by the UK Biotechnology and Biological Sciences Research Council (BBSRC). N.S.S. is a Royal Society Wolfson Merit Award holder and an EPSRC Established Career Fellow. The authors would like to acknowledge the assistance given by IT Services and the use of the Computational Shared Facility at The University of Manchester and the EPSRC UK National Service for Computational Chemistry Software (NSCCS).

\section{References}

[1] a) M. D. Toney, Biochimica et Biophysica Acta (BBA) - Proteins and Proteomics 2011, 1814, 1405-1406; b) R. Percudani, A. Peracchi, EMBO reports 2003, 4, 850854; c) D. E. Metzler, M. Ikawa, E. E. Snell, J. Am. Chem. Soc. 1954, 76, 648-652; d) M. L. di Salvo, R. Contestabile, M. K. Safo, Biochimica et Biophysica Acta (BBA) Proteins and Proteomics 2011, 1814, 1597-1608; e) D. Dolphin, R. Poulson, O. Avramovic, John Wiley \& Sons, New York., 1986; f) A. C. Eliot, J. F. Kirsch, Ann. Rev. Biochem. 2004, 73, 383-415.

[2] a) E. E. Snell, W. T. Jenkins, J. Cell. Comp. Physiol. 1959, 54, 161-177; b) P. Christen, D. E. Metzler, Wiley, J. \& Sons, 1985, pp. 37-101; c) V. N. Malashkevich, M. D. Toney, J. N. Jansonius, Biochemistry 1993, 32, 13451-13462; d) N. Watanabe, M. N. G. James, Biochimica et Biophysica Acta (BBA) - Proteins and Proteomics 2011, 1814, 1528-1533.

[3] a) X. Zhou, M. D. Toney, Biochemistry 1998, 38, 311-320; b) Y.-l. Lin, J. Gao, Biochemistry 2010, 49, 84-94; c) E. J. Fogle, M. D. Toney, Biochemistry 2010, 49, 6485-6493; d) A. A. Moya-García, D. Rodríguez-Agudo, H. Hayashi, M. A. Medina, J. L. Urdiales, F. Sánchez-Jiménez, J. Chem. Theory Comput. 2011, 7, 1935-1942.

[4] a) M. A. Spies, M. D. Toney, Biochemistry 2003, 42, 5099-5107; b) Y.-L. Lin, J. Gao, A. Rubinstein, D. T. Major, Biochimica et Biophysica Acta (BBA) - Proteins and Proteomics 2011, 1814, 1438-1446; c) H. Wolosker, Biochimica et Biophysica Acta (BBA) - Proteins and Proteomics 2011, 1814, 1558-1566; d) H. Wolosker, K. N. Sheth, M. Takahashi, J.-P. Mothet, R. O. Brady, C. D. Ferris, S. H. Snyder, Proc. Natl. Acad. Sci. USA 1999, 96, 721-725.

[5] a) V. Schirch, D. M. E. Szebenyi, Curr. Opin. Chem. Biol. 2005, 9, 482-487; b) S. Raboni, S. Bettati, A. Mozzarelli, Cell. Mol. Life Sci. 2009, 66, 2391-2403; c) A. Mozzarelli, S. Bettati, B. Campanini, E. Salsi, S. Raboni, R. Singh, F. Spyrakis, V. P. Kumar, P. F. Cook, Biochimica et Biophysica Acta (BBA) - Proteins and Proteomics 
2011, 1814, 1497-1510; (d) W. M. Rabeh, P. F. Cook, J. Biol. Chem. 2004, 279, 26803-26806.

[6] a) H.-P. Chen, F.-C. Hsui, L.-Y. Lin, C.-T. Ren, S.-H. Wu, Eur. J. Biochem. 2004, 271, 4293-4297; b) H. A. Barker, Annu. Rev. Biochem. 1981, 50, 23-40; c) K. R. Wolthers, S. E. J. Rigby, N. S. Scrutton, J. Biol. Chem. 2008, 283, 34615-34625; d) K. R. Wolthers, C. Levy, N. S. Scrutton, D. Leys, J. Biol. Chem. 2010, 285, 1394213950; e) C. Makins, F. N. Miros, N. S. Scrutton, K. R. Wolthers, Bioorg. Chem. 2012, 40, 39-47; f) H.-P. Chen, S.-H. Wu, Y.-L. Lin, C.-M. Chen, S.-S. Tsay, J. Biol. Chem. 2001, 276, 44744-44750; g) C. Makins, A. V. Pickering, C. Mariani, K. R. Wolthers, Biochemistry 2013, 52, 878-888; h) C.-H. Tseng, C.-H. Yang, H.-J. Lin, C. Wu, H.-P. Chen, FEMS Microbiol. Lett. 2007, 274, 148-153.

[7] a) P. A. Frey, FASEB J. 1993, 7, 662-670; b) P. A. Frey, O. T. Magnusson, Chem. Rev. 2003, 103, 2129-2148; c) W. Wu, K. W. Lieder, G. H. Reed, P. A. Frey, Biochemistry 1995, 34, 10532-10537; d) M. D. Ballinger, P. A. Frey, G. H. Reed, Biochemistry 1992, 31, 10782-10789; e) P. A. Frey, G. H. Reed, Biochim. Biophys. Acta, Proteins and Proteomics 2011, 1814, 1548-1557; f) F. J. Ruzicka, P. A. Frey, J. Phys. Chem. B 2010, 114, 16118-16124; g) S. C. Wang, P. A. Frey, Biochemistry 2007, 46, 1288912895; h) P. A. Frey, Acc. Chem Res 2013, 47, 540-549.

[8] a) Y.-H. Chen, A. N. Maity, P. A. Frey, S.-C. Ke, J. Am. Chem. Soc. 2013, 135, 788794; b) F. Berkovitch, E. Behshad, K.-H. Tang, E. A. Enns, P. A. Frey, C. L. Drennan, Proc. Natl. Acad. Sci. U. S. A. 2004, 101, 15870-15875; c) C. H. Chang, P. A. Frey, J. Biol. Chem. 2000, 275, 106-114; d) K.-H. Tang, A. D. Casarez, W. Wu, P. A. Frey, Arch. Biochem. Biophys. 2003, 418, 49-54; e) Y.-H. Chen, A. N. Maity, Y.-C. Pan, P. A. Frey, S.-C. Ke, J. Am. Chem. Soc. 2011, 133, 17152-17155; f) K.-H. Tang, S. O. Mansoorabadi, G. H. Reed, P. A. Frey, Biochemistry 2009, 48, 8151-8160; g) A. N. Maity, C.-P. Hsieh, M.-H. Huang, Y.-H. Chen, K.-H. Tang, E. Behshad, P. A. Frey, S.-C. Ke, J. Phys. Chem. B 2009, 113, 12161-12163; h) G. M. Sandala, D. M. Smith, L. Radom, J. Am. Chem. Soc. 2006, 128, 16004-16005; i) A. Maity, Y.-H. Chen, S.-C. Ke, Int. J. Mol. Sci. 2014, 15, 3064-3087.

[9] D. P. Dowling, A. K. Croft, C. L. Drennan, Annu. Rev. Biophys. 2012, 41, 403-427.

[10] a) C. H. Chang, M. D. Ballinger, G. H. Reed, P. A. Frey, Biochemistry 1996, 35, 11081-11084; b) S. D. Wetmore, D. M. Smith, L. Radom, J. Am. Chem. Soc. 2001, 123, 8678-8689.

[11] J. Pang, X. Li, K. Morokuma, N. S. Scrutton, M. J. Sutcliffe, J. Am. Chem. Soc. 2012, 134, 2367-2377.

[12] a) S. D. Wetmore, D. M. Smith, L. Radom, J. Am. Chem. Soc. 2000, 122, 1020810209; b) D. M. Smith, B. T. Golding, L. Radom, J. Am. Chem. Soc. 1999, 121, 57005704; c) D. M. Smith, B. T. Golding, L. Radom, J. Am. Chem. Soc. 1999, 121, 93889399; d) D. M. Smith, B. T. Golding, L. Radom, J. Am. Chem. Soc. 1999, 121, 10371044; e) G. M. Sandala, D. M. Smith, L. Radom, Acc. Chem Res 2010, 43, 642-651.

[13] B. W. Lepore, F. J. Ruzicka, P. A. Frey, D. Ringe, Proc. Natl. Acad. Sci. U. S. A. 2005, 102, 13819-13824.

[14] a) S. Sharif, M. C. Huot, P. M. Tolstoy, M. D. Toney, K. H. M. Jonsson, H.-H. Limbach, J. Phys. Chem. B 2007, 111, 3869-3876; b) H.-H. Limbach, M. Chan-Huot, S. Sharif, P. M. Tolstoy, I. G. Shenderovich, G. S. Denisov, M. D. Toney, Biochimica et Biophysica Acta (BBA) - Proteins and Proteomics 2011, 1814, 1426-1437.

[15] a) S. Sharif, G. S. Denisov, M. D. Toney, H.-H. Limbach, J. Am. Chem. Soc. 2007, 129, 6313-6327; b) S. Sharif, D. Schagen, M. D. Toney, H.-H. Limbach, J. Am. Chem. Soc. 2007, 129, 4440-4455; c) S. Sharif, G. S. Denisov, M. D. Toney, H.-H. Limbach, J. Am. Chem. Soc. 2006, 128, 3375-3387. 
[16] a) X. Li, L. W. Chung, P. Paneth, K. Morokuma, J. Am. Chem. Soc. 2009, 131, 51155125; b) D. Bucher, G. M. Sandala, B. Durbeej, L. Radom, D. M. Smith, J. Am. Chem. Soc. 2012, 134, 1591-1599; c) A. R. Jones, S. J. O. Hardman, S. Hay, N. S. Scrutton, Angew. Chem. Int. Ed. 2011, 50, 10843-10846; d) W. D. Robertson, M. Wang, K. Warncke, J. Am. Chem. Soc. 2011, 133, 6968-6977.

[17] D. T. Major, J. Gao, J. Am. Chem. Soc. 2006, 128, 16345-16357.

[18] a) D. T. Major, K. Nam, J. Gao, J. Am. Chem. Soc. 2006, 128, 8114-8115; b) Y.-l. Lin, J. Gao, J. Am. Chem. Soc. 2011, 133, 4398-4403; c) J. Crugeiras, A. Rios, E. Riveiros, J. P. Richard, J. Am. Chem. Soc. 2011, 133, 3173-3183; d) J. P. Richard, T. L. Amyes, J. Crugeiras, A. Rios, Curr. Opin. Chem. Biol. 2009, 13, 475-483; e) A. Rubinstein, D. T. Major, Biochemistry 2010, 49, 3957-3964; f) R. Casasnovas, M. Adrover, J. Ortega-Castro, J. Frau, J. Donoso, F. Muñoz, J. Phys. Chem. B 2012, 116, 1066510675.

[19] K. P. Jensen, U. Ryde, J. Am. Chem. Soc. 2005, 127, 9117-9128.

[20] A. Watanabe, T. Yoshimura, B. Mikami, H. Hayashi, H. Kagamiyama, N. Esaki, J. Biol. Chem. 2002, 277, 19166-19172.

[21] M. J. Frisch, G. W. Trucks, H. B. Schlegel, G. E. Scuseria, M. A. Robb, J. R. Cheeseman, G. Scalmani, V. Barone, B. Mennucci, G. A. Petersson, H. Nakatsuji, M. Caricato, X. Li, H. P. Hratchian, A. F. Izmaylov, J. Bloino, G. Zheng, J. L. Sonnenberg, M. Hada, M. Ehara, K. Toyota, R. Fukuda, J. Hasegawa, M. Ishida, T. Nakajima, Y. Honda, O. Kitao, H. Nakai, T. Vreven, J. Montgomery, J. A. , J. E. Peralta, F. Ogliaro, M. Bearpark, J. J. Heyd, E. Brothers, K. N. Kudin, V. N. Staroverov, R. Kobayashi, J. Normand, K. Raghavachari, A. Rendell, J. C. Burant, S. S. Iyengar, J. Tomasi, M. Cossi, N. Rega, N. J. Millam, M. Klene, J. E. Knox, J. B. Cross, V. Bakken, C. Adamo, J. Jaramillo, R. Gomperts, R. E. Stratmann, O. Yazyev, A. J. Austin, R. Cammi, C. Pomelli, J. W. Ochterski, R. L. Martin, K. Morokuma, V. G. Zakrzewski, G. A. Voth, P. Salvador, J. J. Dannenberg, S. Dapprich, A. D. Daniels, Ö. Farkas, J. B. Foresman, J. V. Ortiz, J. Cioslowski, D. J. Fox, Gaussian 09, Revision B.1, Gaussian, Inc., Wallingford CT, 2009.

[22] a) J. C. Gordon, J. B. Myers, T. Folta, V. Shoja, L. S. Heath, A. Onufriev, Nucleic Acids Res., 2005, 33, W368-W371; b) J. Myers, G. Grothaus, S. Narayanan, A. Onufriev, Proteins: Struct., Funct., Bioinf. 2006, 63, 928-938; c) R. Anandakrishnan, B. Aguilar, A. V. Onufriev, Nucleic Acids Res. 2012, 40, W537-W541.

[23] a) H. Li, A. D. Robertson, J. H. Jensen, Proteins: Struct., Funct., Bioinf. 2005, 61, 704-721; b) D. C. Bas, D. M. Rogers, J. H. Jensen, Proteins: Struct., Funct., Bioinf. 2008, 73, 765-783; c) M. H. M. Olsson, C. R. Søndergaard, M. Rostkowski, J. H. Jensen, J. Chem. Theory Comput. 2011, 7, 525-537.

[24] W. D. Cornell, P. Cieplak, C. I. Bayly, I. R. Gould, K. M. Merz, D. M. Ferguson, D. C. Spellmeyer, T. Fox, J. W. Caldwell, P. A. Kollman, J. Am. Chem. Soc. 1995, 117, 5179-5197.

[25] a) S. Dapprich, I. Komáromi, K. S. Byun, K. Morokuma, M. J. Frisch, J. Mol. Struct. (Theochem) 1999, 462, 1-21; b) T. Vreven, K. S. Byun, I. Komáromi, S. Dapprich, J. A. Montgomery, K. Morokuma, M. J. Frisch, J. Chem. Theory Comput. 2006, 2, 815826. 


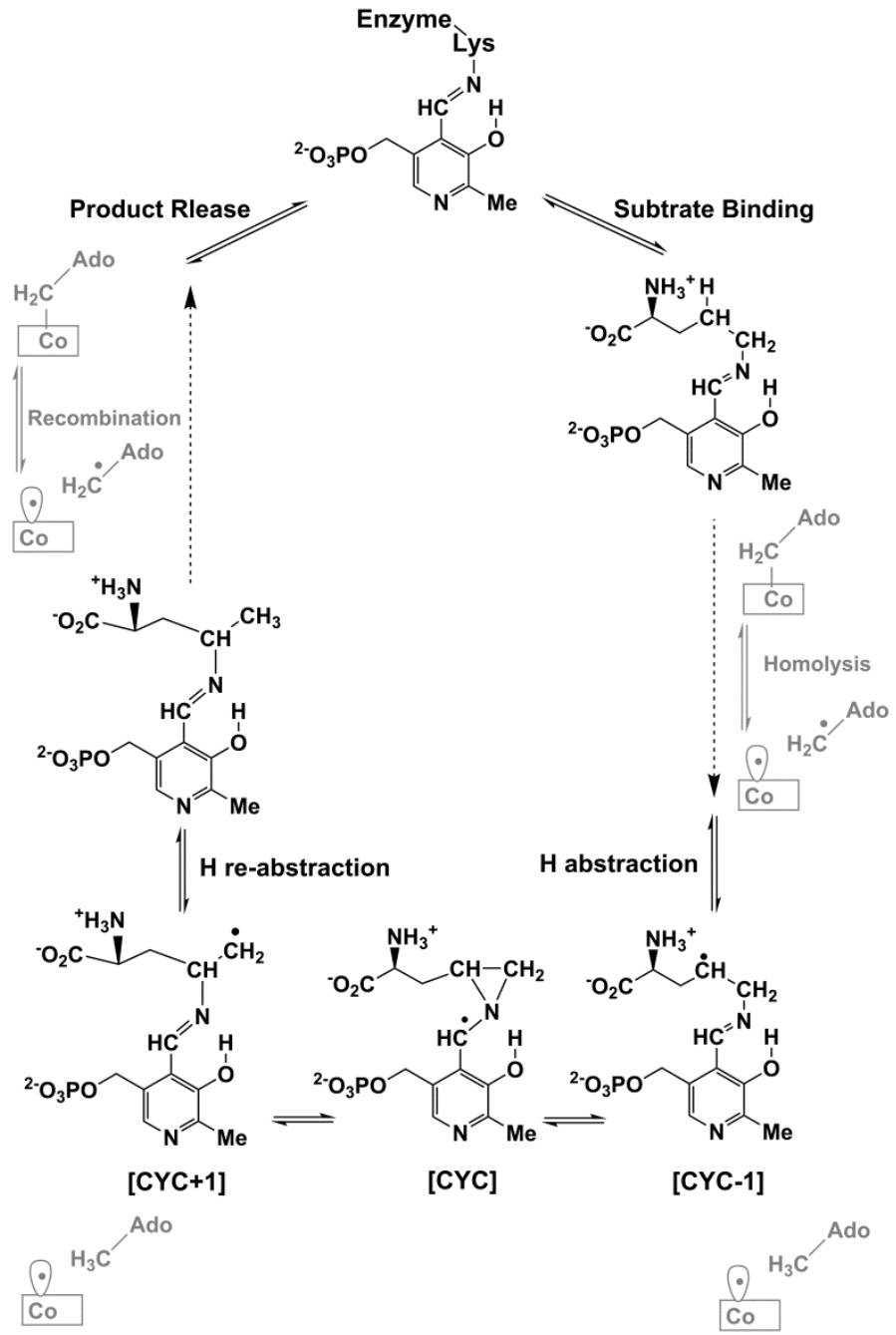

Figure 1: The proposed reaction mechanism for the reaction catalysed by OAM with Dornithine as the substrate (adapted from references ${ }^{[6 c]}$ and ${ }^{[11]}$ ). The intermediates associated with the cyclisation step are labelled as CYC-1, CYC and CYC+1. 


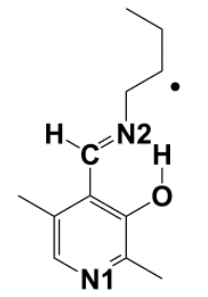

Model_1

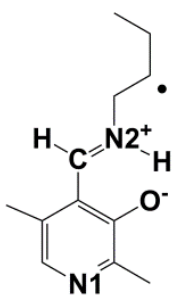

Model_2

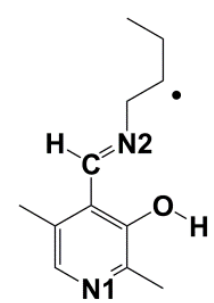

Model_3<smiles></smiles>

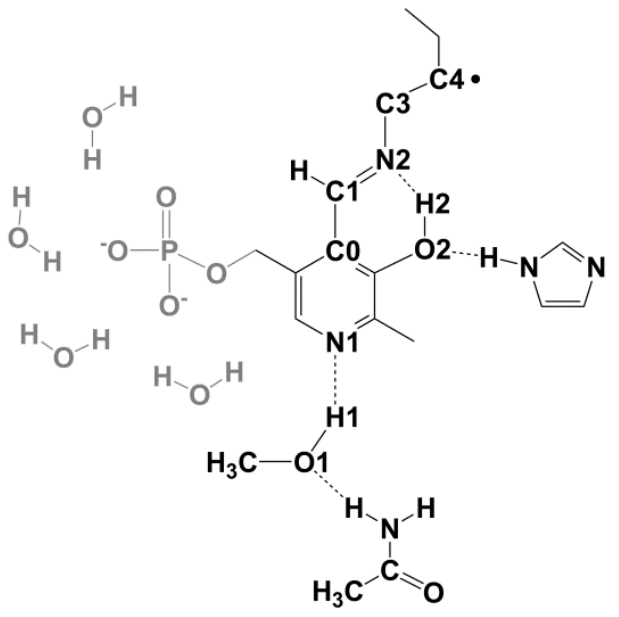

Model_4 and Model_5

Figure 2: Gas phase models. Two sets of models have been constructed to determine the effect of the protonation states of N1 and N2 in PLP. The first set is with the pyridine N1 in the neutral form (Model_1, Model_2 and Model_3, first row). The second set is with N1 protonated (labelled with the suffix $\left(\mathrm{H}^{+}\right)$, second row). Within each set, $\mathrm{O} 2-\mathrm{H} 2{ }^{\cdots} \mathrm{N} 2$ is in the neutral form in Model_1, and in the zwitterionic form in Model_2, while O2-H2 ${ }^{\cdots} \mathrm{N} 2$ is in the neutral firm but with H2 pointing away from O1 in Model_3. Model_4 (shown in black) and Model_5 (shown in black and grey) have been constructed to study the role of Ser162, His225 and the phosphate group. The methanol group is used to mimic the sidechain of Ser162. The imidazole ring is to represent the sidechain of His225. Sidechain of a glutamine is included to mimic the hydrogen bond between Ser162 and Gln183. To study the effect of the phosphate group, Model_4 is built with no phosphate group and Model_5 with the phosphate group and three water molecules to mimic the hydrogen bonds within the active site. 


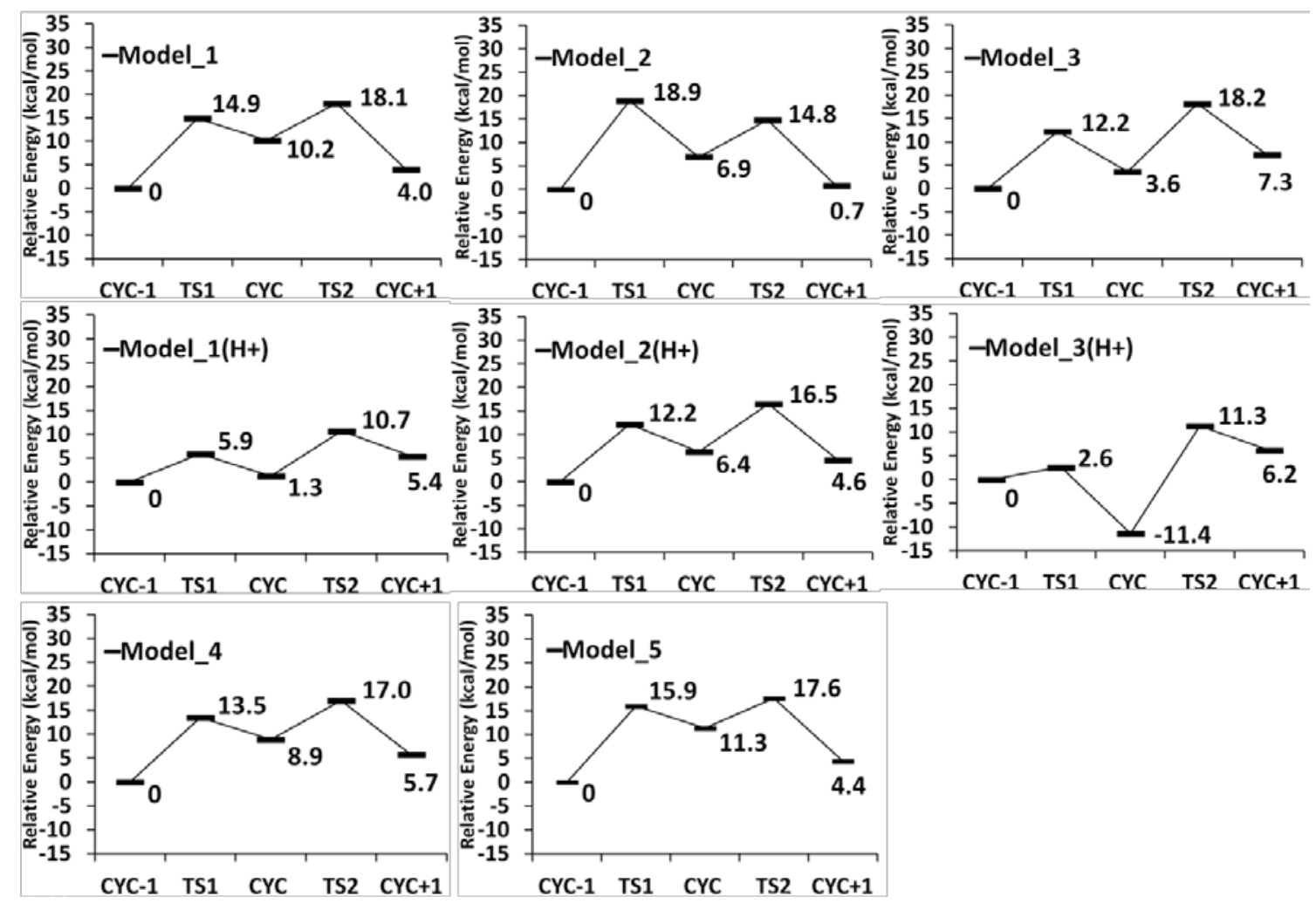

Figure 3: Schematic representation of the barrier height and energy of ring-closing (CYC$1-\mathrm{TS} 1-\mathrm{CYC})$ and ring-opening $(\mathrm{CYC}-\mathrm{TS} 2-\mathrm{CYC}+1)$ for the gas phase models. 


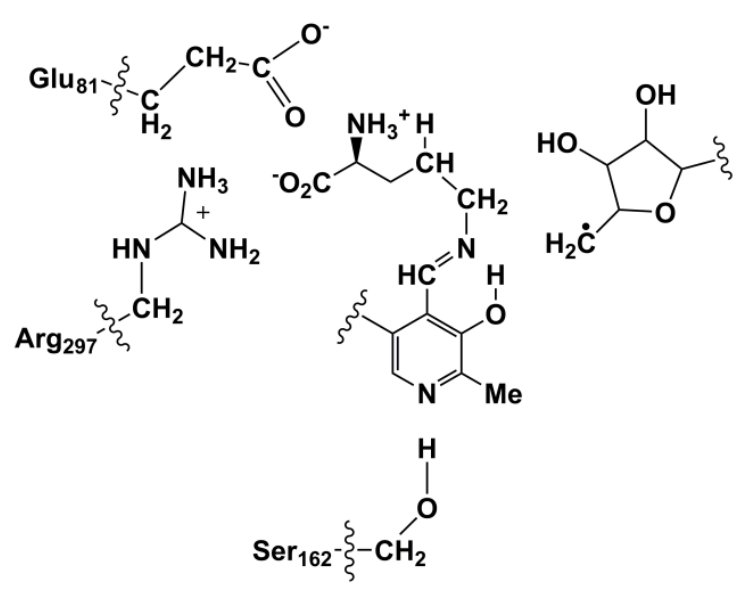

QM/MM_1

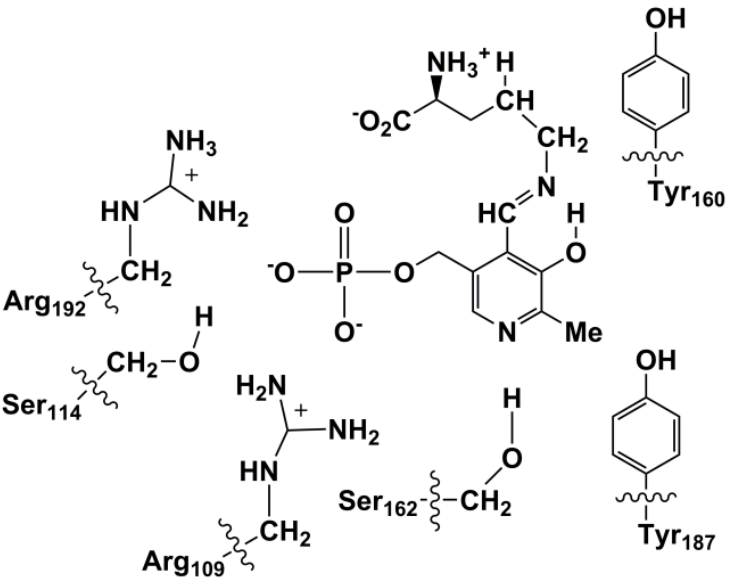

QM/MM_2

Figure 4: Atoms treated quantum mechanically in the ONIOM QM/MM calculations. The QM and MM boundaries are illustrated by the curly lines. The QM region of QM/MM_1 includes the ribose ring of the AdoCbl, the PLP-bound substrate without the phosphate group and the side chains of Ser162, Arg297 and Glu81. The QM region of QM/MM_2 contains the full PLP-bound substrate, including the phosphate group and active site residues Arg109, Ser114, Tyr160, Ser162, Tyr187 and Arg192. Tyr160 and Tyr187 stack above and below the pyridine ring of PLP, forming $\pi-\pi$ interactions. 


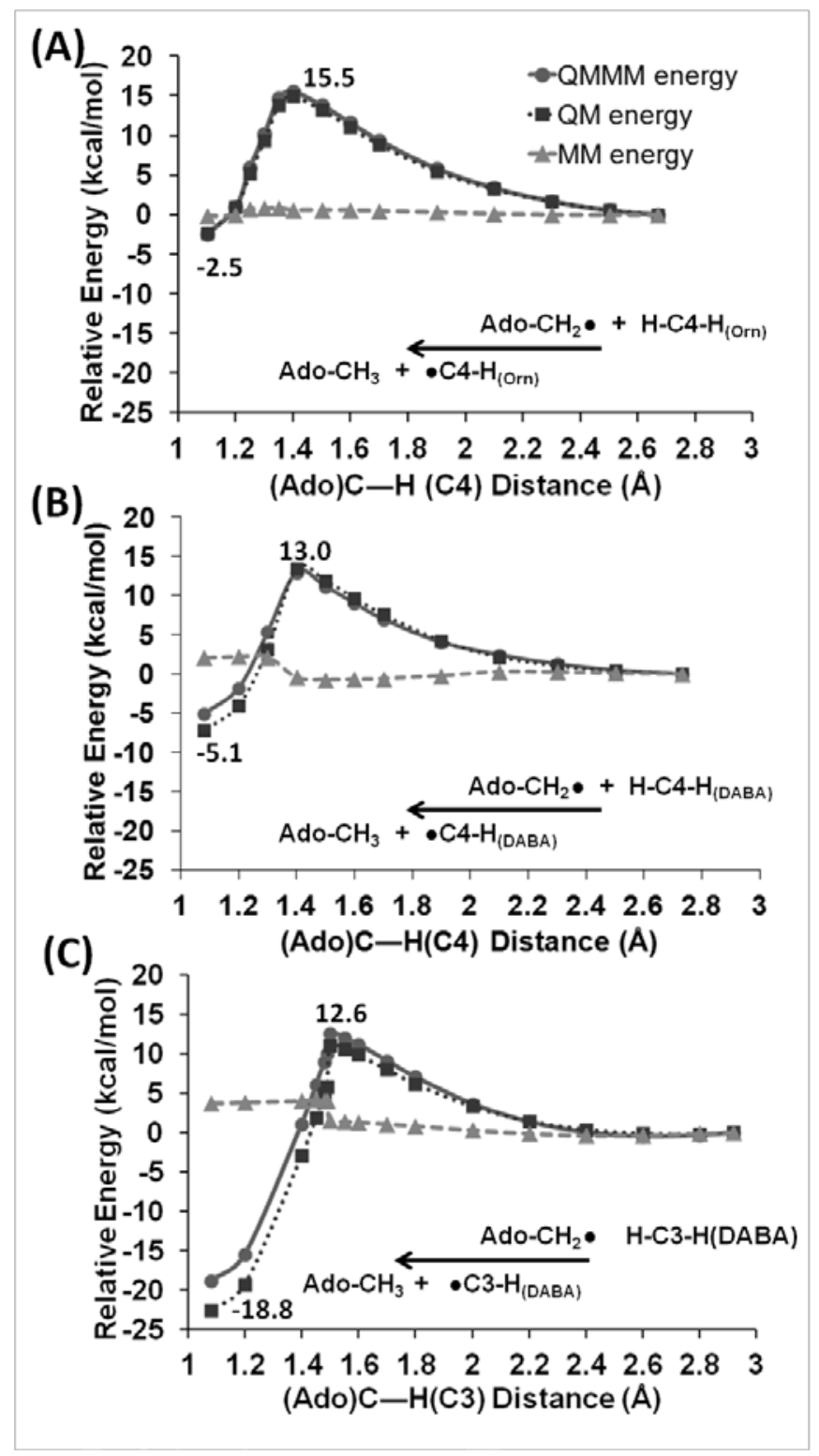

Figure 5: Potential energy profile of the hydrogen abstraction to produce CYC-1 of the PLPbound substrate in $\mathrm{QM} / \mathrm{MM} 1^{M E_{-} E E}(\mathrm{~A})$, the C4-radical of the PLP-bound DABA in QM/MM_DABA ${ }^{M E \_E E}$ (B) and the C3-radical of the PLP-bound DABA in $\mathrm{QM} / \mathrm{MM} \_D A B A^{M E \_E E}(\mathrm{C})$. The energies are given relative to the right hand side (the $5^{\prime}$ deoxyadenosyl radical, Ado- $\mathrm{CH}_{2} \bullet$ ). The barrier height and the energy of reaction of the QM/MM energy are labelled. The initial distances between the hydrogen to be abstracted and the Ado- $\mathrm{CH}_{2} \bullet$ radical range from $\sim 2.7$ to $\sim 2.9 \AA$. 

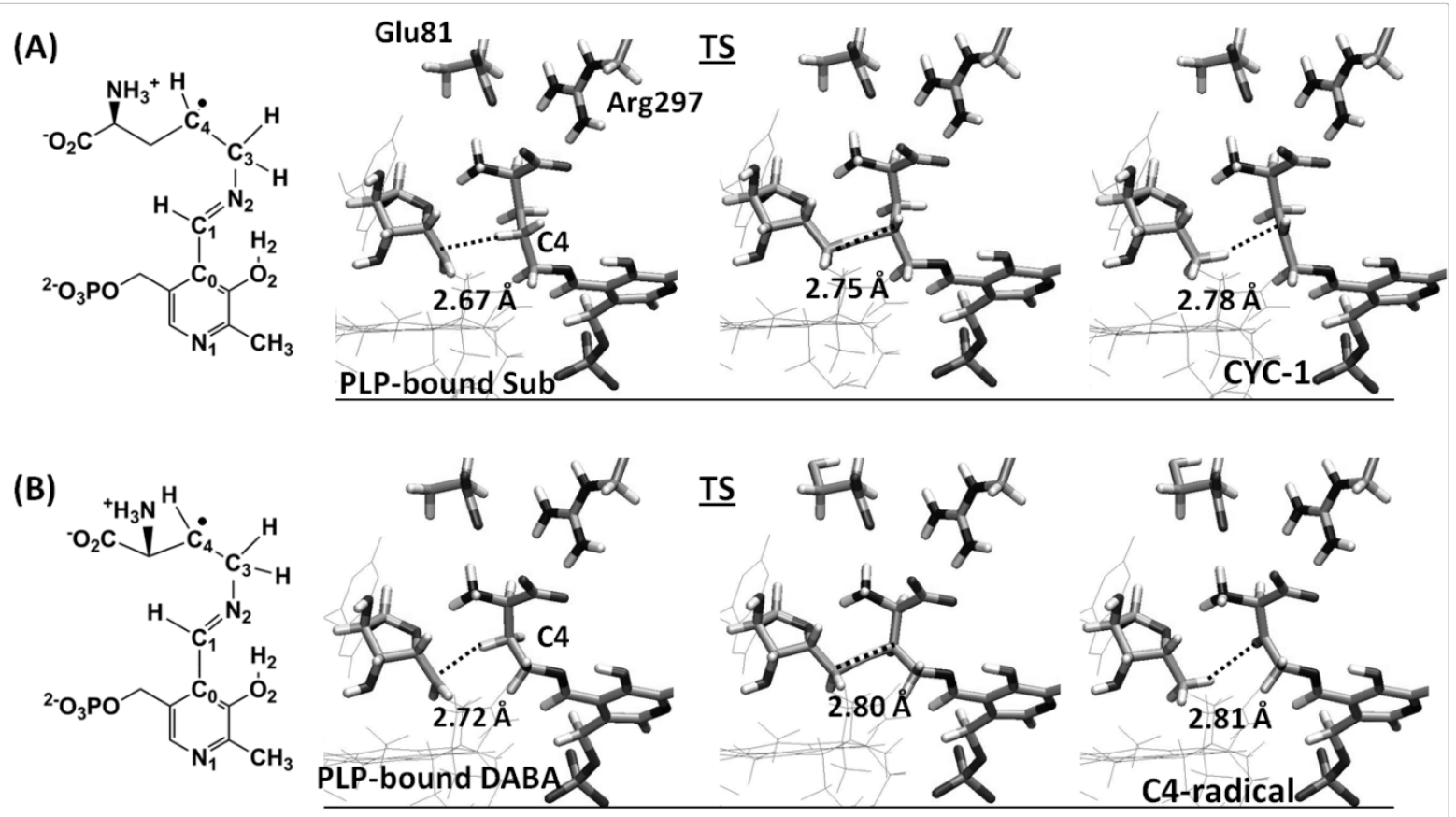

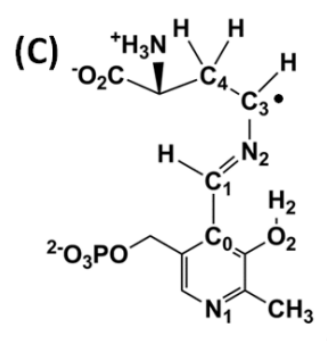
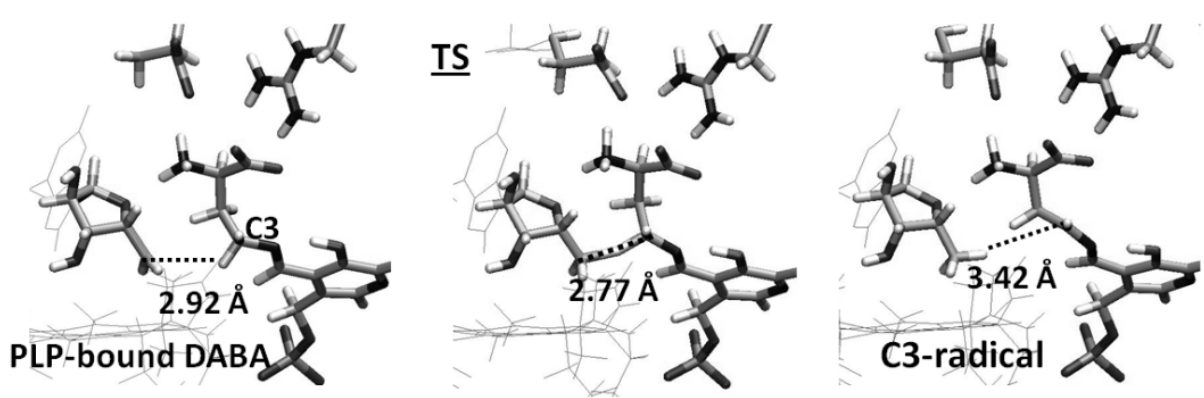

Figure 6: Geometry associated with the hydrogen abstraction to produce CYC-1 of the PLPbound substrate (A), the C4-radical of the PLP-bound DABA (B) and the C3-radical of the PLP-bound DABA (C). Active site residues Arg297 and Glu81 are also displayed. The labelling of atoms is shown on the left hand side of each panel to aid interpretation of the structures. 


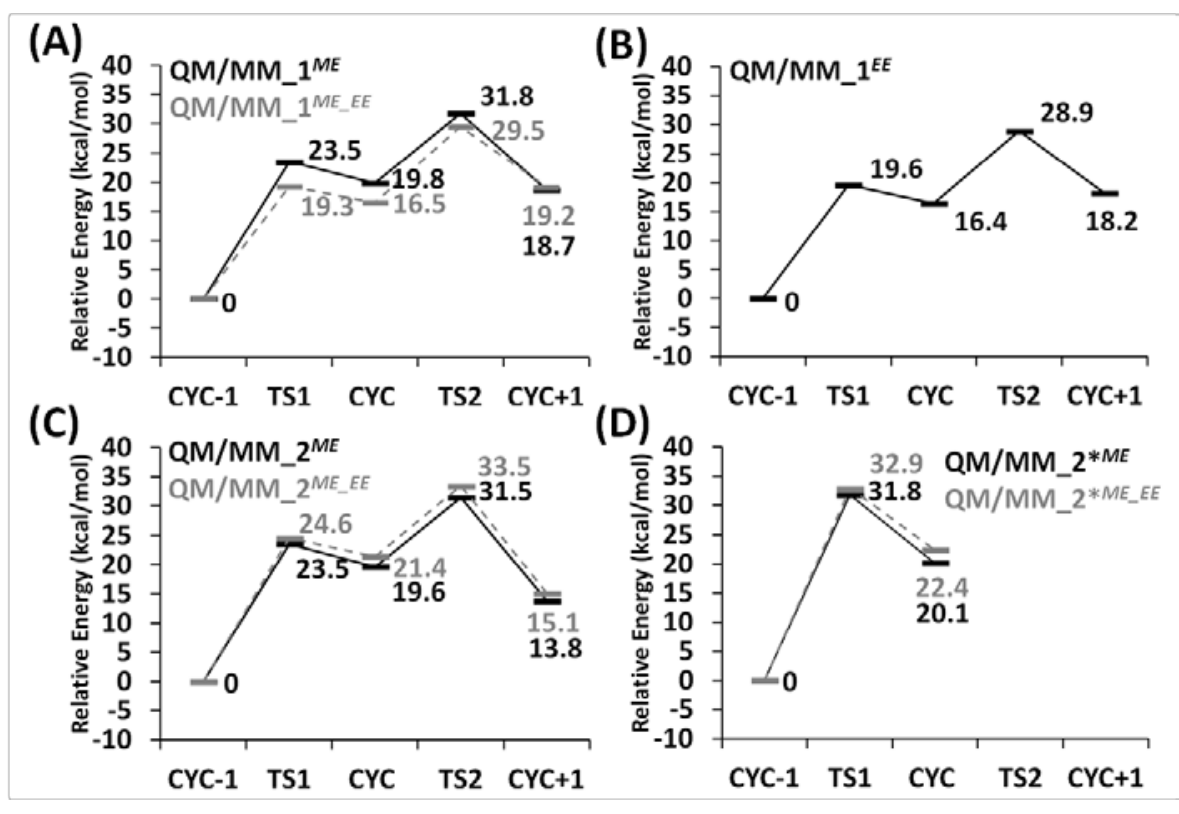

Figure 7: Schematic representation of the barrier height and energy of ring-closing (CYC$1-\mathrm{TS} 1-\mathrm{CYC})$ and ring-opening $(\mathrm{CYC}-\mathrm{TS} 2-\mathrm{CYC}+1)$ from the QM/MM calculations. (A) and (B) are from QM/MM_1 while (C) and (D) are from QM/MM_2. The superscripts "ME” and "EE" indicate the application of ME scheme and EE scheme in geometry optimisation, respectively, while the superscript "ME_EE" indicates the energy from single point calculation with the EE scheme based on the ME-optimised geometry. QM/MM_2* corresponds to QM/MM_2 with the $\mathrm{O} 2-\mathrm{H} 2{ }^{\cdots} \mathrm{N} 2$ in the zwitterionic form. 

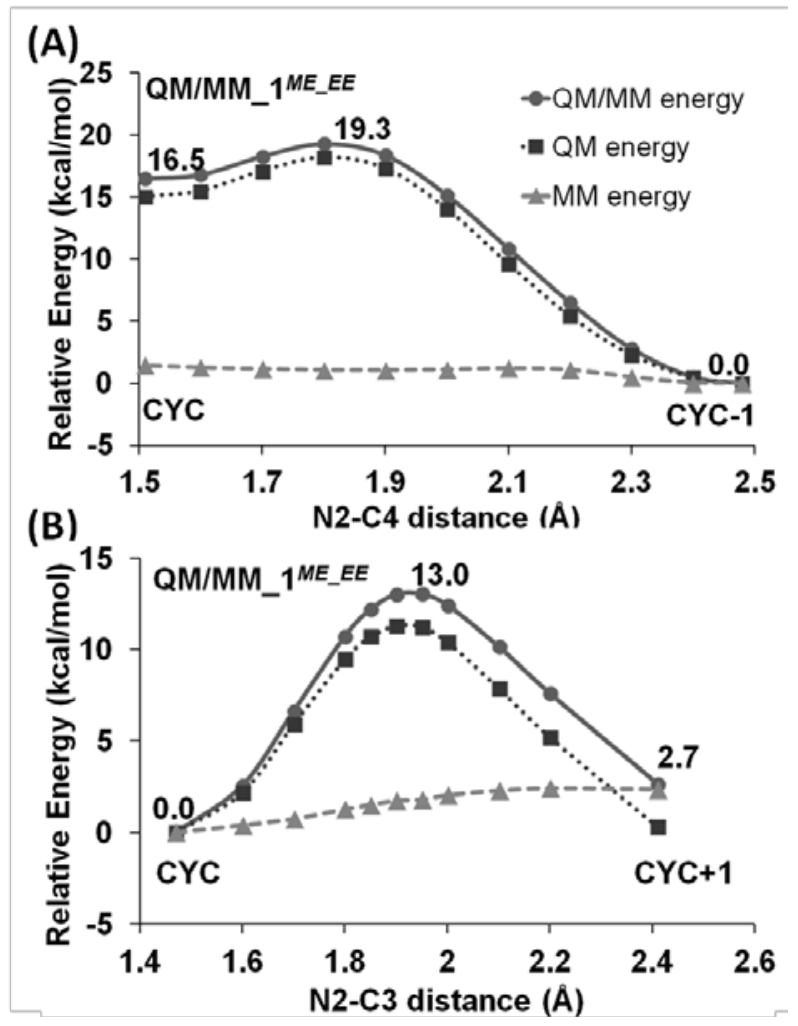

Figure 8: Energy decomposition of the QM/MM energy of (A) ring-closing (CYC-1-CYC) and $(\mathrm{B})$ ring-opening $(\mathrm{CYC}-\mathrm{CYC}+1)$ from $\mathrm{QM} / \mathrm{MM} 1^{M E_{-} E E}$. The energies are given relative to CYC-1 (N2-C4 distance $2.48 \AA$ ) in (A) and are given relative to CYC (N2-C3 distance $1.47 \AA$ ) in (B). 

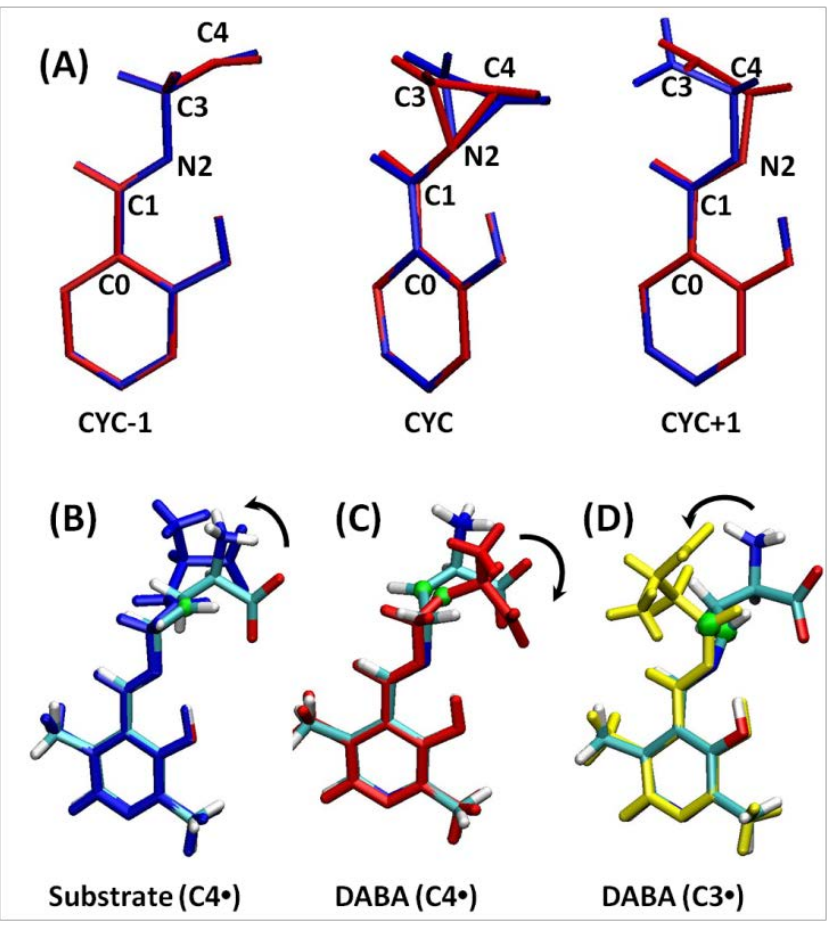

Figure 9: (A) Overlay of the structures of CYC-1, CYC and CYC+1 from Model_1 (blue) and QM/MM_1 (red) with the relevant atoms labelled. For clarity, only the pyridine ring and hydrogens involved in the cyclisation steps are displayed. See Table S1 in the SI for a comprehensive list of dihedral angles associated with these structures. (B), (C) and (D) Overlay of the structures from gas phase and the enzyme, from which the "strain" energy is calculated. Figure (B) is CYC-1 from the PLP-bound substrate while Figures (C) and (D) are the C4 and C3 radicals derived from the PLP-bound DABA. The gas phase structure is shown in blue, red and yellow, respectively. The structures in the enzyme are displayed with the usual colouring scheme. The radical carbons are highlighted in green. Overlaid structures were obtained by superimposing the pyridine ring. 


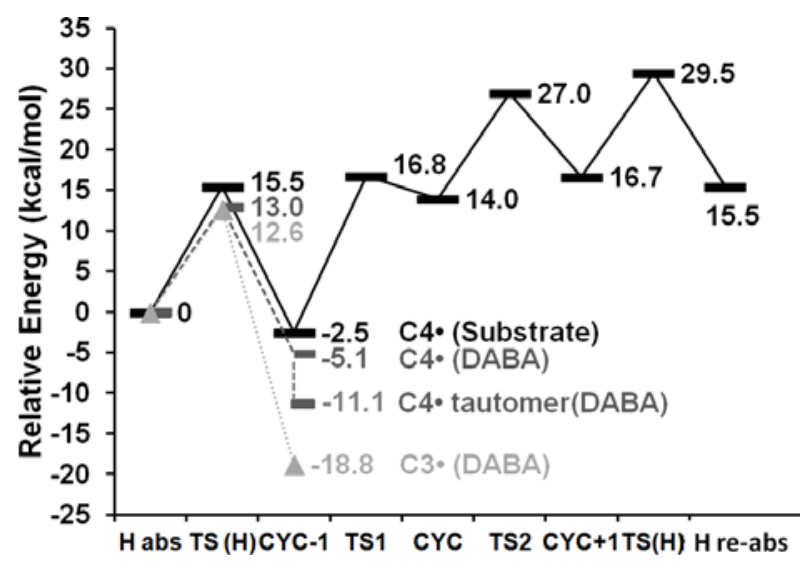

Figure 10: Potential energy profile of the hydrogen abstraction ( $\mathrm{H}$ abs-TS(H) - CYC-1), ring-closing (CYC-1-TS1-CYC), ring-opening (CYC-TS2-CYC+1) and the hydrogen re-abstraction $\left(\mathrm{CYC}+1-\mathrm{TS}(\mathrm{H})-\mathrm{H}\right.$ re-abs) for the substrate ornithine (QM/MM_1 ${ }^{M E} \_E E$, black solid line). The potential energy profile of the hydrogen abstraction from C3 and C4 of DABA (QM/MM_DABA ${ }^{M E \_E E}$ ) is shown in lighter grey with dotted line and darker grey with dashed line, respectively. 
Table 1: Summary of the barrier height and energy of reaction of ring-closing (CYC-1 to $\mathrm{CYC}$ ) and ring-opening (CYC to CYC+1) for the gas phase models and the QM/MM systems (See Figure 2 and 4 for the structure and definition of the systems). Numbers in the brackets are the energy of ring-opening $(\mathrm{CYC}-\mathrm{TS} 2-\mathrm{CYC}+1)$ given relative to $\mathrm{CYC}$. The superscripts "ME" and "EE" indicate the application of the mechanical embedding (ME) scheme and electronic embedding (EE) scheme in the optimisation, while the superscript "ME_EE" indicates the energy from single point calculation with the EE scheme based on the ME-optimised geometry. QM/MM_2* is QM/MM_2 with O2-H2*N2 in the zwitterionic form. The energy is given in $\mathrm{kcal} / \mathrm{mol}$.

\begin{tabular}{|c|c|c|c|c|c|}
\hline & CYC-1 & TS1 & CYC & TS2 & $\mathrm{CYC}+1$ \\
\hline Model_1 & 0 & 14.9 & $10.2(0)$ & 18.1 (7.9) & $4.0(-6.2)$ \\
\hline Model_2 & 0 & 18.9 & $6.9(0)^{a}$ & $14.8(7.9)^{a}$ & $0.7(-6.2)^{a}$ \\
\hline Model_3 & 0 & 12.2 & $3.6(0)$ & $18.2(14.6)$ & 7.3 (3.7) \\
\hline Model_1( $\left.\mathrm{H}^{+}\right)$ & 0 & 5.9 & $1.3(0)$ & $10.7 \quad(9.4)$ & $5.4 \quad(4.1)$ \\
\hline Model_2(H+) & 0 & 12.2 & $6.4(0)$ & $16.5(10.1)$ & $4.6(-1.8)$ \\
\hline Model_3( $\left.\mathrm{H}^{+}\right)$ & 0 & 2.6 & $-11.4(0)$ & 11.3 (22.7) & $6.2(17.6)$ \\
\hline Model_4 & 0 & 13.5 & $8.9(0)$ & $17.0 \quad(8.1)$ & $5.7(-3.2)$ \\
\hline Model_5 & 0 & 15.9 & $11.3(0)$ & $17.6 \quad(6.3)$ & $4.4(-6.9)$ \\
\hline $\mathrm{QM} / \mathrm{MM} \_1^{M E}$ & 0 & 23.5 & $19.8(0)$ & $31.8(12.0)$ & $18.7(-1.1)$ \\
\hline $\mathrm{QM} / \mathrm{MM} \_1^{M E_{-} E E}$ & 0 & 19.3 & $16.5(0)$ & $29.5(13.0)$ & $19.2(2.7)$ \\
\hline $\mathrm{QM} / \mathrm{MM} \_1^{E E}$ & 0 & 19.6 & $16.4(0)$ & $28.9(12.5)$ & $18.2(1.8)$ \\
\hline $\mathrm{QM} / \mathrm{MM} \_2^{M E}$ & 0 & 23.5 & $19.6(0)$ & 31.5 (11.9) & $13.8(-5.8)$ \\
\hline $\mathrm{QM} / \mathrm{MM} \_2^{M E \_E E}$ & 0 & 24.6 & $21.4(0)$ & $33.5(12.1)$ & $15.1(-6.3)$ \\
\hline $\mathrm{QM} / \mathrm{MM} \_2^{* M E}$ & 0 & 31.8 & 20.1 & -- & -- \\
\hline $\mathrm{QM} / \mathrm{MM} \_2^{* M E \_E E}$ & 0 & 32.9 & 22.4 & -- & -- \\
\hline
\end{tabular}

* In QM/MM_2*, upon formation of CYC, O2...H2-N2 is optimised back to the neutral state during the potential energy scan to allow a path of lower potential energy to be followed. 
Table 2: The computed hyperfine coupling parameters for ${ }^{2} \mathrm{H}(\mathrm{C} 1)$ of the PLP-bound DABA derived radicals. The hyperfine coupling parameters are the sum of the isotropic and anisotropic coupling parameters. The unit is MHz. See Figure S8 in the SI for the structure of the C4 tautomer.

\begin{tabular}{|c|c|c|c|c|c|c|}
\hline & & & \multicolumn{4}{|c|}{$A\left({ }^{2} \mathrm{H}\right)_{\text {ENDOR }}(\mathrm{MHz})$} \\
\hline & Radical & ${ }^{2} \mathrm{H}$ label & & $A_{\mathrm{xx}}$ & $A_{\mathrm{yy}}$ & $A_{\mathrm{zz}}$ \\
\hline \multirow{8}{*}{ Calculation } & \multirow[t]{2}{*}{ C3 } & \multirow[t]{2}{*}{${ }^{2} \mathrm{H}(\mathrm{C} 1)$} & Gas Phase & -7.29 & -5.02 & -2.03 \\
\hline & & & Enzyme & -7.85 & -5.30 & -1.94 \\
\hline & \multirow[t]{2}{*}{ C4 } & \multirow[t]{2}{*}{${ }^{2} \mathrm{H}(\mathrm{C} 1)$} & Gas Phase & -0.45 & -0.30 & 0.12 \\
\hline & & & Enzyme & -0.17 & -0.15 & 0.63 \\
\hline & \multirow[t]{4}{*}{ C4 tautomer } & \multirow{2}{*}{${ }^{2} \mathrm{H}_{\mathrm{b} 1}(\mathrm{C} 1)$} & Gas Phase & 7.99 & 8.32 & 9.25 \\
\hline & & & Enzyme & 7.55 & 7.65 & 8.68 \\
\hline & & \multirow{2}{*}{${ }^{2} \mathrm{H}_{\mathrm{b} 2}(\mathrm{C} 1)$} & Gas Phase & 6.04 & 6.51 & 7.34 \\
\hline & & & Enzyme & 0.87 & 1.30 & 2.13 \\
\hline Experiment* & & & & -4.80 & -5.00 & -8.10 \\
\hline
\end{tabular}

* The experimental ENDOR data is taken from 5,6-LAM with DABA as the substrate. ${ }^{[8 a]}$ 


\section{Entry for the Table of Contents}

Layout 1:

Radicals in a PLP-dependent reaction

Dr Jiayun Pang, * Prof. Nigel S. Scrutton and Prof. Michael J. Sutcliffe

Combined QM/MM Studies of the Mechanism of Action of Cofactor Pyridoxal 5'-Phosphate in Ornithine 4,5-Aminomutase

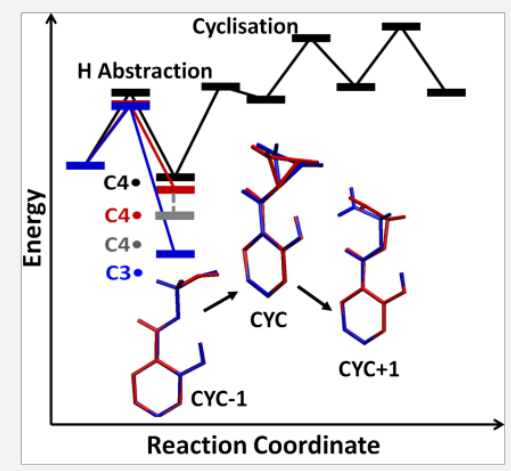

The experimentally elusive hydrogen abstraction and cyclisation steps in the PLP-dependent, D-ornithine 4,5aminomutase (OAM)-catalysed reaction is studied with a $\mathrm{QM} / \mathrm{MM}$ approach. Substantial "strain” effect of the active site is imposed on the orientation of the cyclic intermediate in order to control its trajectory and may also play a key role in differentiating radical species derived from the substrate and its analogues.

KEYWORDS: enzyme catalysis, radical reactions, PLP, ONIOM(DFT:MM), aminomutase 